\title{
Physio-chemical hydrodynamic mechanism underlying the formation of thin adsorbed boundary films
}

\author{
W. W. F. Chong ${ }^{1}$, M. Teodorescu ${ }^{1,2}$ and H. Rahnejat ${ }^{3}$ \\ ${ }^{1}$ School of Engineering, Cranfield University, Cranfield, UK \\ ${ }^{2}$ Baskin School of Engineering, University of California at Santa Cruz, CA USA \\ 3 Wolfson School of Mechanical \& Manufacturing Engineering, Loughborough \\ University, Loughborough, UK \\ E-mail: mteodorescu@soe.ucsc.edu
}

\begin{abstract}
.
Formation of low shear strength surface-adhered thin films mitigates excessive friction in mixed or boundary regimes of lubrication. Tribo-films are formed as a consequence of molecular chemical reaction with the surfaces. The process is best viewed in the context of a lubricant-surface system. Therefore, it is usually surmised that the adsorption of lubricant molecular species to the contact surfaces would be underlying to the formation of ultra-thin lubricant films.

The paper considers contact of smooth surfaces at close separation. This may be regarded as the contact of a pair of asperity summits, whose dimensions, however small, are far larger than the size of fluid molecules within the conjunction. In such diminishing separations the constraining effect of relatively smooth solid barriers causes oscillatory solvation of fluid molecules. This effect accounts for the conjunctional load capacity but does not contribute to mitigating friction, except when molecular adsorption is taken into account with long chain molecules which tend to inhibit solvation. The paper presents an analytical predictive model based on the Ornstein-Zernike method with Percus-Yevick approximation of a narrow interaction potential between conjunctional composition. The predictions confirm the above stated physical facts in a fundamental manner.
\end{abstract}




\section{Introduction}

Except for some occasions where friction is required for functional assurance such as locomotion and traction, in most other circumstances its reduction would improve energy efficiency. As far back as the 17th century the interaction of rough surface topography was understood to be the main underlying mechanism for friction both at the onset of motion (Amontons [1]) and in its pursuance (De Coulomb [2]). Indeed there is compelling evidence that separation of rough surfaces by a film of low shear strength was intuitively understood even in the ancient times. Historical records show the use of water or vegetable oil between wooden sleds and wooden tracks or tree trunks, for example in the bas relief in the grotto of El Bersheh and vegetable grease or even mud in the bearings of the chariot of Ur in Mesopotamia.

In the 17th Century, Newton [3] described the slow viscous action in relative motion of hard spherical fluid molecules giving rise to shear resistance, which he used to define the concept of viscosity. This was the first tacit inference that like solids, fluids also give rise to friction, enabling tractive motion. Later the concept of viscosity and internal fluidic friction led to the works of Navier [4], Poisson [5] and Stoke [6] and culminated in the fundamental equation for fluid flow (the Navier-Stoke equations). In the turn of 20th century Reynolds [7] modified the Navier-Stoke equations for narrow conjunctions where the effect of body, inertial and surface forces may be ignored. Reynolds [7] assumed the formation of a coherent film of lubricant between a pair of loaded contiguous surfaces in relative motion.

In hydrodynamics, the film thickness is inversely proportional to the applied load. It subsequently became apparent that a coherent film cannot be attained at all instances. The absence of wear in such circumstances was viewed as puzzling by Reynolds [7], with ever predicted decreasing film thickness. This in time led to the understanding of piezoviscous action of lubricants, simultaneous with the localised elastic deflection of solids, noted many years earlier by Hertz [8]. The elastohydrodynamic regime of lubrication (EHL) was first declared by Grubin [9]. The key feature of EHL is the insensitivity of lubricant film thickness to increasing load.

In the turn of 20th century it was often noted that the break-down or breach of a coherent lubricant film does not lead to friction conditions pertaining to dry contact of solid boundaries in the form of dry friction which was noted by Coulomb [2]. In particular, Petrov [10] proposed a different form of lubricant viscosity: "stickiness", which he proposed was due to the preferential positioning of some molecules near surfaces. Following the same argument, Hardy and Doubleday [11] stated that "another kind of lubrication" exists when the solid faces are near enough to directly influence the chemistry of lubricants. They termed this as boundary lubrication. Hardy and Doubleday [11] noted the low shear strength of these chemical films.

As Dowson [12] has observed, the thickness of fluid films has spectacularly reduced since the time of Reynolds, where it may have been of the order of tens to hundreds of micrometers. The progressive reduction in film thickness throughout the 20th century 
has been partly as the result of miniaturisation of all forms of mechanisms and devices and partly because of a greater understanding of the role of boundary lubrication. Dowson states that talk of films of tenths of micrometer and even nanometers is now commonplace.

In a pioneering paper, Bowden and Tabor [13] showed that a thin film of molecular dimensions can be adsorbed to the topographical surface features, whose summits are large compared with the dimensions of molecules. This confirmed Petrov's earlier supposition that the "stickiness" of the fluids near the surfaces is due to their increased viscosity there. At the relatively smooth summit of asperities the density of molecules is greater than in the bulk of any thin lubricating film as shown, for example by Israelachvilli [14].

An experiment carried out by Chan and Horn [15] with ultra-smooth surfaces at vanishing separation of molecular dimensions showed discrete drainage of fluid molecules in a stepwise fashion from the conjunction. The phenomenon caused by the constraining effect of surfaces and the ensuing interactions with the intervening fluid molecules is known as solvation, where the packing order of molecules several molecular diameter in depth differs from that in the bulk of a fluid film (Israelachvilli [14], Gohar and Rahnejat [16]). These findings are in accord with the suppositions put forward in the 19th century by Petrov [10] and later by Hardy and Doubleday [11]. The generated pressures in such conjunctions are subject to attractive-repulsive solvation effect as the fluid film is successively discretised into rows of molecules which are drained from the conjunction in a stepwise fashion. Matsuoka and Kato [17] and Al-Samieh and Rahnejat $[18,19]$ have shown that in tribological contacts where the entrainment of a fluid film is through hydrodynamic action by relative motion of surfaces, the solvation effect only dominates in gaps of several molecular diameter. They have also shown that its effect supersedes any van der Waals interaction between the fluid molecules and between those and the atoms of the bounding surfaces. Mutual approach of surfaces, promoting hydrodynamics in squeeze film motion was also found to inhibit molecular layering (Teodorescu et al [20]) as well as the introduction of micro-meniscus effect, which sets the limit of adhesion at conjunction depths of the order of two molecular diameters of a non-polar mono-molecular idealised fluid (Al-Samieh and Rahnejat [21]). Using the same approach, in a recent contribution Chong et al [22] considered idealised fluids of mono-molecular composition represented as spherical particles with different molecular diameters. The long chain molecules were represented with smaller crosssectional diameters. The examples used were Octamethycyclotetrasiloxane (OMCTS), an additive of silicone oil and Hexadecane: a long chain thin molecule used as an additive boundary lubricant species. It was shown that the effect of solvation was significantly attenuated with the Hexadecane when compared with OMCTS. This was in accord with the findings of Israelachvilli [14]. The long chain molecules reduced the load carrying capacity, but improved friction. The converse was found to be the case for small spherical molecules of OMCTS. The study of Chong et al [22] also included hydrodynamic entrainment of the fluid into the conjunction of a pair of asperity summits, 
where the combined effect of hydrodynamics, solvation and van der Waals interaction accounted for the load carrying capacity. The effect of lubricant cavitation and inlet starvation were also taken into account.

All these contributions have used the empirical relationship proposed by Israelachvilli [14] for generated solvation pressures, which do not take into account the effect of molecular adsorption to the solid surfaces. Therefore, Petrov's so-called molecular "stickiness" and its effect upon shear characteristics of the lubricant was neglected. They also employed idealised fluids of mono-molecular composition. The study by Chong et al [22] showed that shear characteristics of the contact closely resembled viscous shear of a thin lubricant film, with step-wise increases in shear as a form of work required to eject a layer of molecules from the conjunction.

Real fluids comprise a mix of molecular species in certain concentrations and packing fraction. The former corresponds to the concentration of various species. In particular, boundary lubricants as additive species represent a small proportion of the bulk lubricant volume; referred to as the base oil. The latter; packing fraction, acknowledges the state of the fluidic medium, with progressively lower dispositions corresponding to vapour or gaseous media. Chong et al [23] extended their mono-molecular model to bi-molecular mixture, with the spherical additive molecules possessing adsorption energy to stick to the bounding surfaces. The model is based on the solution of Ornstein and Zernike [24] (OZ) integral equation, with an infinitely narrow attractive well potential represented by the Percus and Yevick [25] (PY) approximation. An analytical physio-chemical model was thus established which unlike molecular dynamics can be extended to broader contacting regions with significantly reduced computation.

It is natural to extend this work to hard long chain molecules, which is more representative of real boundary lubricant additive species. The Ornstein and Zernike [24] method can also be used to model the structure, molecular correlation, and thermodynamic properties of chain-like fluids [26, 27, 28, 29, 30, 31]. For the hard chain spherical molecules, the model proposed by Baxter [32] is employed at the assumed sticky end of a long chain. This is the approach highlighted in the current study.

\section{Mathematical Model}

As the initial investigation, a real fluid comprising two molecular species is assumed. One of these is a hard spherical molecule with no adsorption, as an allusion to the base lubricant molecular species. In practice oils have several species constituting the base oil. The other in a small percentage is a long chain molecule used as an additive, particularly as friction modifier for boundary lubrication. Model of a lubricant as a real fluid should comprise such a composition. A basic model is shown in figure 1, where both types of molecule are shown. They interact with each other as well as with the atoms of the bounding surfaces.

The interaction of hard molecules, confined by planar walls, can be modelled using 
the hard sphere model of Perram and Smith [33] (figure 1). For long chain molecules, the concept of "sticky" hard spheres proposed by Baxter [32] is used, where adsorption (as the underlying mechanism for "stickiness") is assumed to occur at one end of the molecular chain. Furthermore, for molecular chains a connectivity constraint must be imposed (Chiew [34, 35, 36]).

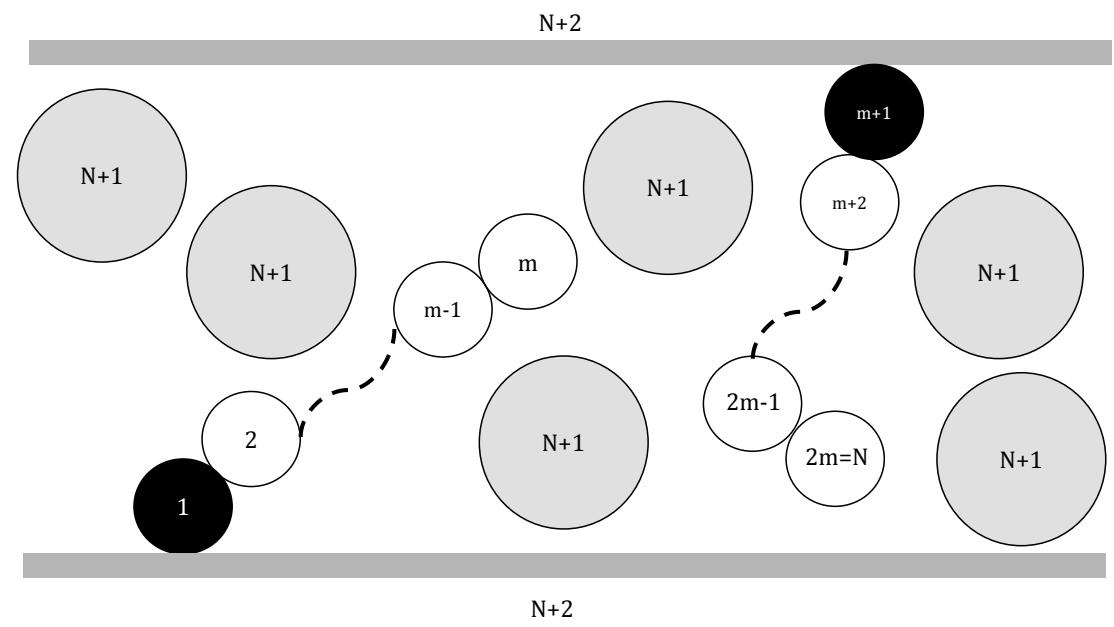

Figure 1. Fluid mixtures of spheres/chains of $m$-mer with one end of the chain (represented by particle 1) adsorbing to the surface

\subsection{Hard Spheres}

Initially, the fluid between two surfaces is modelled as a large number of interacting hard spheres $[37,17,32]$. These are impenetrable particles of diameter, $\sigma_{\alpha}$ and $\sigma_{\beta}$ with a centre-to-centre distance of $r^{*}$ (figure 2). To represent long chain molecules hard spheres can be linked via the connectivity constraints [34, 35, 36] (section 2.2).

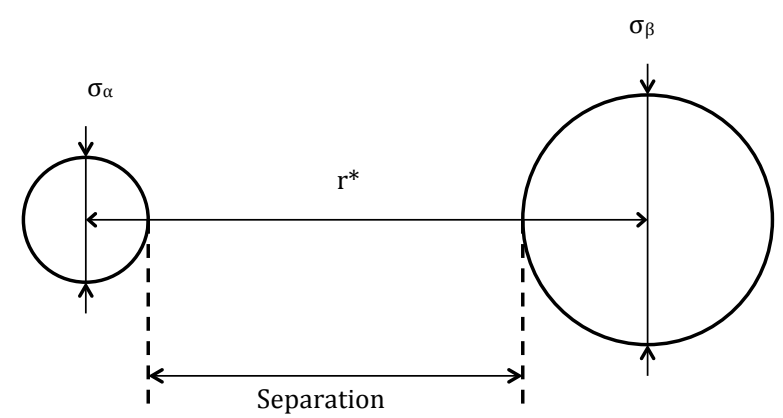

Figure 2. Interaction between a pair of particles

The solvation pressure between approaching surfaces arises as a result of the fluid particles being constrained in a diminishing gap of the same scale as their molecular size. Using the Derjaguin approximation $[14,38]$ for hard spheres confined by macroscopic flat bodies, the solvation pressure, $p_{s}$ becomes: 


$$
p_{s}=-\frac{d^{2}}{d r^{* 2}} \frac{1}{2 \pi R_{e f f}}\left[k_{B} T\left(g^{*}\left(r^{*}\right)-1\right)\right]
$$

where $R_{e f f}=\sigma_{\alpha} \sigma_{\beta} / 2\left(\sigma_{\alpha}+\sigma_{\beta}\right)$ is the effective diameter, $k_{B}$ is the Boltzmann constant, $T$ is the temperature of the fluid system, $\alpha$ and $\beta$ refer to the hard spherical species and $g^{*}\left(r^{*}\right)$ is the pair correlation function at distance $r^{*}$ (described later).

The pair correlation function $\left(g_{\alpha \beta}^{*}\right.$ in equation (1)) describes the probability of finding a particle of species $\beta$ at a distance, $r^{*}$ from a referenced particle: species $\alpha$. The particles (spheres) interact through central forces, thus the pair correlation function, $g_{\alpha \beta}^{*}$ is expressed as:

$$
g_{\alpha \beta}^{*}\left(r^{*}\right)=h_{\alpha \beta}^{*}\left(r^{*}\right)+1, \quad \alpha, \beta \in\{1,2,3 \ldots(N+2)\}
$$

where $h_{\alpha \beta}^{*}$ is the indirect correlation function, which describes the indirect influence of a particle of species $\alpha$ on another particle of species $\beta$ through an intervening particle, which can be either of species $\alpha$ or $\beta$.

Ornstein and Zernike [24] proposed the following equation for the indirect correlation function $\left.\left(h_{\alpha \beta}^{*}\right)\right)$ :

$$
h_{\alpha \beta}^{*}\left(r^{*}\right)=c_{\alpha \beta}^{*}+\sum_{\gamma=1}^{N+2} \rho_{\gamma}^{*} \int c_{\alpha \gamma}^{*}(|s|) h_{\gamma \beta}^{*}\left(\left|r^{*}-s\right|\right) d s
$$

where $(N+2)$ is the total number of hard spherical species and $s$ is the distance between any particle and a reference particle, where $r^{*}$ is the centre-to-centre spacing. The direct correlation function, $c_{\alpha \beta}^{*}$ determines the interaction between the spherical particles of species $\alpha$ and $\beta$. The parameter $\rho_{\gamma}^{*}$ refers to the number density or particle density for the hard spheres of species $\gamma \in\{\alpha, \beta\}$.

The Percus-Yevick [25] (PY) approximation (equation (4)) is used in this study rather than the Convolution-Hypernetted Chain (CHNC) approximation, because the latter often causes divergence in solutions of multi-molecular fluids [39]. Additionally, PY approximation is simpler to apply and suitable for strongly repulsive interactions (e.g. solvation) leads to accurate results [40]:

$$
c_{\alpha \beta}^{*}\left(r^{*}\right)=g_{\alpha \beta}^{*} \cdot\left(r^{*}\right)\left[1-e^{\phi_{\alpha \beta}\left(r^{*}\right) / k_{B} T}\right]
$$

The attractive potential $\phi_{\alpha \beta}$ in equation (4) describes the adsorption between molecules $\alpha$ and $\beta$ [41]. The adsorption refers to the adhesion of particles, which form a molecularly-thin layer, adhered to a surface. Baxter [32] describes the adsorption of molecules to surfaces based on infinitesimally short range potentials. He solves the Ornstein-Zernike equation analytically, using the Percus-Yevick approximation (OZ-PY) for a single component fluid. Perram and Smith [33] extended the work of Baxter to a 
fluid mixture. They defined the infinitely narrow well potential for a fluid mixture, $\phi_{\alpha \beta}$ as:

$$
\phi_{\alpha \beta}(r) / k_{B} T= \begin{cases}\log \left[12 \bar{T}_{\alpha \beta} \delta / R_{\alpha \beta}\right] & r^{*} \leq R_{\alpha \beta} \\ 0 & r^{*}>R_{\alpha \beta}\end{cases}
$$

where $R_{\alpha \beta}=\left(\sigma_{\alpha}+\sigma_{\beta}\right) / 2, \bar{T}_{\alpha \beta}$ is the dimensionless temperature of the fluid and $\delta$ is the Dirac delta function, defining the limit of the infinitely narrow well potential. The adsorbent-adsorbate attraction described in this approach is characterised by a single energy parameter. Therefore, the particles coming into direct contact with the adsorbate planar surface are deemed as adsorbed.

Assuming $c_{\alpha \beta}^{*}=0$ as $r^{*}>R_{\alpha \beta}$ and using Wiener-Hopf factorisation [42], equation (3) becomes [41, 43]:

$$
\begin{aligned}
r^{*} h_{\alpha \beta}^{*}\left(\left|r^{*}\right|\right)= & -\frac{d}{d r^{*}} q_{\alpha \beta}\left(r^{*}\right) \\
& +2 \pi \sum_{\gamma=1}^{N+2} \rho_{\gamma}^{*} \int_{S_{\alpha \gamma}}^{R_{\alpha \gamma}} q_{\alpha \gamma}(s)\left(r^{*}-s\right) h_{\gamma \beta}^{*}\left(\left|r^{*}-s\right|\right) d s
\end{aligned}
$$

where: $S_{\alpha \beta}=\left(\sigma_{\alpha}-\sigma_{\beta}\right) / 2$ and

A) $S_{\alpha \beta}<r^{*}<R_{\alpha \beta}$,

$$
q_{\alpha \beta}\left(r^{*}\right)=a_{\alpha}\left(r^{* 2}-R_{\alpha \beta}^{2}\right) / 2+b_{\alpha}\left(r^{*}-R_{\alpha \beta}\right)+\lambda_{\alpha \beta} R_{\alpha \beta}^{2} / 12
$$

where

$$
\begin{aligned}
& a_{\alpha}=\left(1-\zeta_{3}+3 R_{\alpha} \zeta_{2}\right) /\left(1-\zeta_{3}\right)^{2}-X_{\alpha} /\left(1-\zeta_{3}\right) \\
& b_{\alpha}=\left(-3 R_{\alpha}^{2} \zeta_{2}\right) / 2\left(1-\zeta_{3}\right)^{2}-R_{\alpha} X_{\alpha} / 2\left(1-\zeta_{3}\right) \\
& X_{\alpha}=\frac{\pi}{6} \sum_{\gamma=1}^{N+2} \rho_{\gamma}^{*} \lambda_{\alpha \gamma} R_{\alpha \gamma}^{2} \sigma_{\gamma} \\
& \zeta_{j}=\frac{\pi}{6} \sum_{\gamma=1}^{N+2} \rho_{\gamma}^{*} \sigma_{\gamma}^{j}, \quad j=1,2,3
\end{aligned}
$$

B) $r^{*}>R_{\alpha \beta}$,

$$
q_{\alpha \beta}\left(r^{*}\right)=\frac{d}{d r^{*}} q_{\alpha \beta}\left(r^{*}\right)=0
$$

Assuming planar or flat surfaces to be species $(N+2)$, particle diameter $\sigma_{N+2} \rightarrow \infty$ and the number density or particle density $\rho_{N+2}^{*} \rightarrow 0$, equation (6) can be now written as: 


$$
\begin{aligned}
r^{*} h_{\alpha \beta}^{*}\left(\left|r^{*}\right|\right)= & -\frac{d}{d r^{*}} q_{\alpha \beta}\left(r^{*}\right) \\
& +2 \pi \sum_{\gamma=1}^{N+1} \rho_{\gamma}^{*} \int_{S_{\alpha \gamma}}^{R_{\alpha \gamma}} q_{\alpha \gamma}(s)\left(r^{*}-s\right) h_{\gamma \beta}^{*}\left(\left|r^{*}-s\right|\right) d s
\end{aligned}
$$

The $\lambda_{\alpha \beta}$ parameter can be related to the dimensionless temperature of the system $\bar{T}$ using the approximation proposed by Perram and Smith [43]. The relationship can be written as follows:

$$
\lambda_{\alpha \beta} \bar{T}_{\alpha \beta}=a_{\alpha}+b_{\alpha} / R_{\alpha \beta}+\frac{\pi}{6} \sum_{\gamma=1}^{N+2} \rho_{\gamma} \frac{\lambda_{\beta \gamma} R_{\beta \gamma}^{2}}{R_{\alpha \beta}} q_{\alpha \gamma}\left(S_{\alpha \gamma}\right)
$$

The hard spheres are initially considered not to adhere/adsorb to each other but only to the bounding solid wall $[40,43,44]$. The assumption provides a solution to $\lambda_{\alpha, N+2}(\alpha \in\{1,2,3 \ldots(N+2)\})$. The sphere-to-sphere interaction, forming the hard chain is discussed in section 2.2.

With the wall species diameter, $\sigma_{N+2} \rightarrow \infty$, the adsorption parameter, $d_{\alpha}$ can be defined as [40]:

$$
d_{\alpha}(T)=\int_{0}^{\infty}\left[e^{-\phi_{\alpha}\left(r^{*}\right) / k_{B} T}-1\right] d r^{*}
$$

The corresponding infinitely narrow well potential, $\phi_{\alpha}$ for hard spheres adsorbing to the planar wall in equation (11) is [40]:

$$
\phi_{\alpha}\left(r^{*}\right)= \begin{cases}-\epsilon\left(\sigma_{\alpha}-r^{*}\right) / \sigma_{\alpha} & 0<r^{*}<\sigma_{\alpha} \\ 0 & r^{*}>\sigma_{\alpha}\end{cases}
$$

where $\epsilon$ is the adsorption energy per unit $k_{B} T$. Substituting $a_{\alpha}, b_{\alpha}$ and $S_{\alpha}$ into equation (10), $\lambda_{\alpha, M+1}$ can be determined using the following expression [40]:

$$
\frac{\lambda_{\alpha, N+2} R_{\alpha, N+2}}{12 d_{\alpha}(T)}=\frac{1-\zeta_{3}+3 \sigma_{\alpha} \zeta_{2}}{\left(1-\zeta_{3}\right)^{2}}-\frac{\pi \sigma_{\alpha}}{12\left(1-\zeta_{3}\right)} \sum_{\gamma=1}^{N+1} \rho_{\gamma}^{*} \sigma_{\gamma} \lambda_{\gamma, N+2} R_{\gamma, N+2}
$$

The pair correlation function, $g_{\alpha \beta}^{*}$ can be calculated once the indirect correlation function, $h_{\alpha \beta}^{*}$ is evaluated using equation (9). The solvation pressure $p_{s}$ generated by hard spheres confined by flat surfaces is computed from equation (1), using a forward finite difference scheme [17]. 


\subsection{Molecular Chains}

Chiew $[36,34,35]$ note that for a system of mono-molecular hard chain of a fixed length, $m$, the molecular density becomes:

$$
\rho_{c}^{*}=\frac{N_{c}}{V}
$$

where $N_{c}$ is the total number of chains and $V$ is the total volume of the system. The total number of hard spherical species (required to form chain-like molecules) present in the system is:

$$
N=m N_{c}
$$

and the packing fraction of these hard sphere species is

$$
\eta^{*}=\frac{\pi \rho^{*} \sigma^{3}}{6}=\frac{\pi m \rho_{c}^{*} \sigma^{3}}{6}
$$

where $\rho^{*}$ is the number density of the hard spherical species. To represent molecular chains, the $N_{c}$ chain system is viewed as a multi-component particle mixture in which the total number of hard spheres or segments in the chain is $N(=m N c)$. Thus, each particle in a multi-component mixture is regarded as a distinct species (figure 1).

The number density of species $\alpha$ is assumed to be $\rho_{\alpha}^{o}=1 / V$, because there is only one species $\alpha$-type particle in the system. The superscript $o$ denotes the distinct species of spheres forming the chain. Therefore, in the case of $m$-mer chains, as an example, molecule 1 is considered to be formed by particles of species $1,2 \ldots \ldots m$ (figure 1). These constraints for the pair interactions between particles of species $1,2 \ldots \ldots m$, forming molecule 1 is required to fulfil the condition [36]:

$$
\int_{0}^{R_{\alpha \beta}} \rho_{\alpha}^{o} 4 \pi r^{* 2} g_{\alpha \beta}^{*} \cdot d r^{*}=1
$$

where $\beta=\alpha+1$ for $\alpha=1,2 \ldots \ldots m-1$ and $\beta=\alpha-1$ for $\alpha=2,3 \ldots \ldots m$. For $0<r^{*}<R_{\alpha \beta}$, the particle-particle pair correlation function, $g_{\alpha \beta}^{*}$ must take the form

$$
g_{\alpha \beta}^{*}\left(r^{*}\right)=\frac{\lambda_{\alpha \beta}^{o} R_{\alpha \beta}}{12} \delta\left(r^{*}-R_{\alpha \beta}\right)
$$

Equations (16) and (17) require that the parameter $\lambda_{\alpha \beta}^{o}\left(=\lambda_{\alpha \beta}\right.$ in equation (10)) to adopt the following form:

$$
2 \lambda_{\alpha \beta}^{o} \eta_{\beta}^{o}=1
$$

where $\eta_{\beta}^{o}=\pi \rho_{\beta}^{o} \sigma_{\beta}^{3} / 6$ and $\alpha, \beta \neq(N+1)$ and $(N+2)$. For example, the parameters $\lambda_{1, \beta}^{o}=0$ except for $\beta=2$ and so on. 
Equations (16)-(18) describe the connectivity constraints required to form a single hard chain molecule. Similar constraints should also be upheld for the formation of any subsequent chain molecules. For a mixture of chain molecules in a solvent of hard spherical molecules (e.g. base lubricants), the first particles of the $N$-species form the chain of molecules, while particle species $(N+1)$ is the hard sphere solvent. The planar walls are represented by particle species $(N+2)$. As a first approximation, particles of species $i(=1, m+1,2 m+1 \ldots \ldots)$ are assumed to adsorb to the solid barriers. Now, $\lambda_{i, N+2}$ can be computed using equation (12), where $i(=1, m+1,2 m+1 \ldots \ldots)$. The described connectivity constraints can be applied to equations (7)-(10) in order to predict the solvation pressure of a fluid mixture made of hard spheres/molecular chains confined by planar walls.

\section{Conjunctional Friction}

Friction generated by the ultra-thin adsorbed films on nominally molecularly smooth surfaces follow a non-Newtonian shear due to chemical reactions which are based on the thermal activation energy. Eyring [45] developed a model which described the viscosity of fluids under such conditions. It assumes that the motion of a volume of fluid molecules taking place in the presence of a "cage-like" potential barrier by their mere closely- packed arrangement in the bulk. In order to overcome this potential and escape the cage, the fluid molecules strive to surmount the activation barrier potential. This is the thermal activation model which Eyring [45] modified to include the effects of prevailing pressure, $p_{s}$ and shear, $\tau_{y}$ as:

$$
E_{y}=Q_{y}+p_{s} \Omega_{y}-\tau_{y} \phi_{y}^{*}
$$

where $E_{y}$ is the barrier height for the Eyring model, $Q_{y}$ is the process activation energy, $\Omega_{y}$ is the pressure activation volume where pressure, $p_{s}$ is acting and $\phi_{y}^{*}$ is the shear activation volume. The shear activation volume, $\phi_{y}^{*}$ is interpreted as the size of segment that moves during any unit shearing process. This volume can be a part of a molecule or a dislocation line. The pressure activation volume, $\Omega_{y}$ is associated with a local increase in volume to permit the molecular motion to occur.

The applied pressure is due to solvation, as both hydrodynamic and van der Waals pressures are small in comparison. Therefore, the barrier height, $E_{y}$ is equivalent to the solvation energy produced by the hard sphere particles. The barrier height, $E_{y}$ can be expressed as:

$$
E_{y}=\frac{1}{2 \pi R_{e f f}} \cdot \frac{d}{d r^{*}}\left[k_{B} T\left(g^{*}\left(r^{*}\right)-1\right)\right]
$$

By rearranging equation (19), the shear stress, $\tau_{y}$ can be defined as: 


$$
\tau_{y}=\frac{Q_{y}+p_{s} \Omega_{y}-E_{y}}{\phi_{y}^{*}}
$$

The parameters $Q_{y}, \Omega$ and $\phi_{y}^{*}$ must be obtained experimentally. As a first approximation, the values of these parameters are taken from the experimental measurements of He et al [46]. These are: $Q_{y}=1.33 \times 10^{-20} \mathrm{~J}, \Omega_{y}=1.93 \times 10^{-13} \mathrm{~m}^{3} / \mathrm{m}^{2}$ and $\phi_{y}^{*}=1.21 \times 10^{-12} \mathrm{~m}^{3} / \mathrm{m}^{2}$. Therefore, the shear stress between planar walls can be predicted using equation (21).

\section{Results and Discussions}

For the purpose of simulation, hard spheres of $1 \mathrm{~nm}$ diameter are chosen as the base solvent molecules. These are supplemented by spherical chains of nominal diameter $0.5 \mathrm{~nm}$. A total packing fraction of $\eta_{t o t}^{*}$ is assumed for the fluidic volume, constrained within nominally smooth planar walls, representing the flattened contact of a pair of opposing asperities. Therefore, for the fluid mixture, the packing fraction for the long chain and hard spherical molecules becomes:

$$
\begin{aligned}
& \eta_{h s}^{*}=\left(1-r_{w}\right) \eta_{t o t}^{*} \\
& \eta_{h c}^{*}=r_{w} \eta_{t o t}^{*}
\end{aligned}
$$

where $r_{w}$ is the concentration ratio of the hard chain molecules added to the solvent's spherical molecules.

The two bounding solid surfaces are assumed at progressively diminishing separations. For a total packing fraction, $\eta_{t o t}^{*}=0.2$, figure 3 shows the generated solvation pressure and the corresponding shear stress, when the intervening fluid is assumed to comprise molecular chains only. The results in figures 3(a) and (b) discount adsorption potential of the molecules, whilst those in figures 3(c) and (d) include adsorption introduced to only one end of the molecular chains with the adsorption energy per unit $k_{B} T$. With adsorption taken into account a progressively larger number of molecules tend to stick to the bounding surfaces and a thin adsorbed film can result. The pressure increases due to a larger molecular content within the fluidic volume, as solvation attempts to drain layers of molecules from the contact with decreasing separations. This means that adsorption has effectively increased the molecular packing order and an additional energy is required to shear through the adsorbed film. The shear stress may be interpreted to be as the result of a "surface adhered viscosity" or Petrov's [10] "stickiness", but it is far less than that which would occur from direct interaction of rough solid surfaces, thus deviate from dry Coulombic friction as noted by Hardy and Doubleday [11]. It is interesting to note the reduced oscillatory solvation pressure with longer chain molecules, effectively indicating lower load carrying capacity, but reduced boundary friction. 


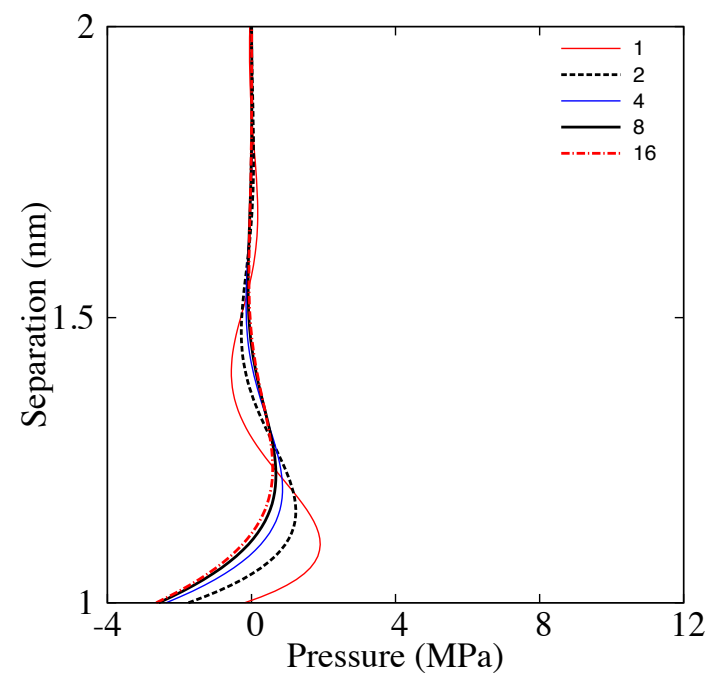

(a) No adsorption

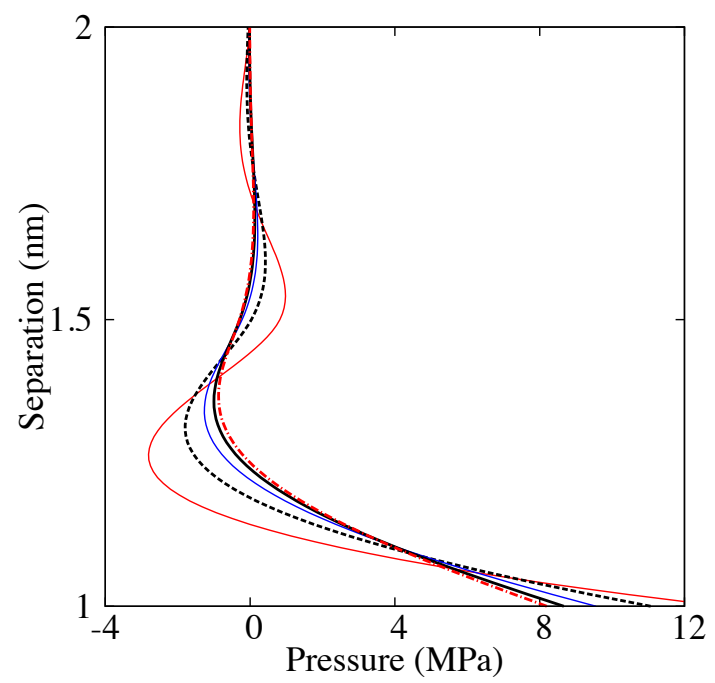

(c) $\epsilon=10$

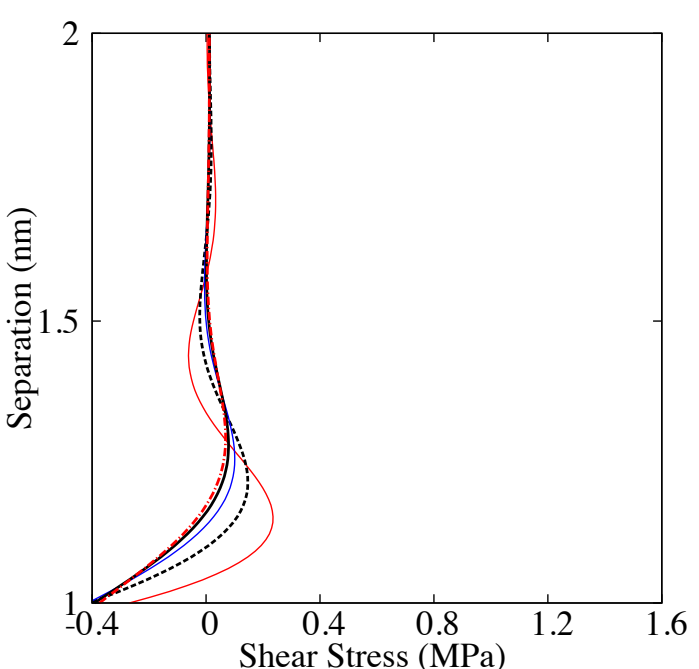

(b) No adsorption

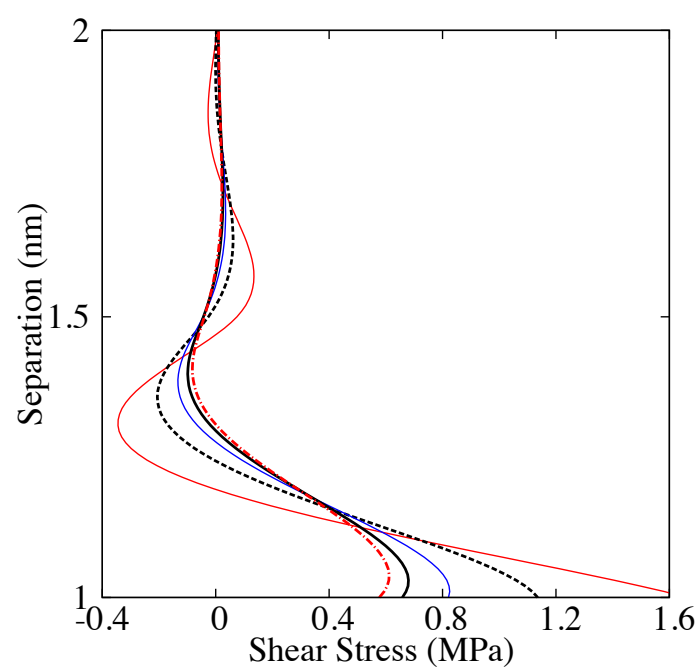

(d) $\epsilon=10$

Figure 3. Contact pressure and shear stress for various molecular chain lengths $(m$ mers) of hard chains confined by planar walls (with/without adsorption) $\left(\eta_{t o t}^{*}=0.2\right)$

As already noted, long chain molecules usually account for a small portion of the volume of lubricant. The case of a fluid with base spherical solvent molecules with no adsorption energy with various concentrations of a 4 -mer chain as the additive is considered next. Figure 4 shows the normalised pressure (the ratio of the interfacial fluid-surface solvation pressure of the mixture to that with zero concentration of the additive molecule) and the corresponding shear characteristics. The adsorption energy of the long chain molecules $(h c)$, in this case represented by 4-mer chains is 100 times larger than the base solvent spherical molecules $(h s)$. Two points merit observation. Firstly, as the packing fraction within the volume increases at the same temperature, the fluidic environment tends to a liquid medium, increasing the number density of 
molecules near the solid barriers, thus enhancing the solvation pressure and the load carrying capacity of the conjunction. At the same time a larger number of long chain molecules adsorb to the boundary solids and form a thin film. Progressively lower packing fractions constitute a trend towards vapour or gaseous dominated media with reduced shear strength, but poorer load capacity.

Secondly, the result indicate that "optimum" conditions may be determined with sufficient load capacity and low shear characteristics by controlling the concentration of $h c$ molecules in the mixture or its adsorption energy (figure 5). Figure 5 shows that for the same concentration of 4-mer hca higher adsorption energy results in a thicker surface adhered film. This constitutes a reduction in shear strength (figure 5(b)). At the same time a narrower conjunction results in which solvation of base molecule induces higher pressure (figure 5(a)). Referring back to figure 4, the results show that some small concentration of $h c$ would be preferred, as indeed is the case in all boundary lubricants; a conclusion arrived at through long term empirical assessment, not because of any scientific rigour. With no concentration of $h c$ molecules, the conjunction behaviour may be seen as that expected of a base fluid (a base lubricant of mono-molecular structure). The difference in shear characteristics between the base fluid and one with small concentrations of the $h c$ additive is more pronounced for lower packing fraction of molecular content. In practice, this means that lower packing fractions should be used, with the significant part of the volume taken up by fillers, anti-oxidants, surfactants and other species, which is a common practice. The other extreme case corresponds to the active molecular mix which is dominated by the $h c$ molecules. This is a rather theoretical scenario as any significant $h c$ content tends to dramatically reduce the load capacity and cause gross slip at boundary solids.

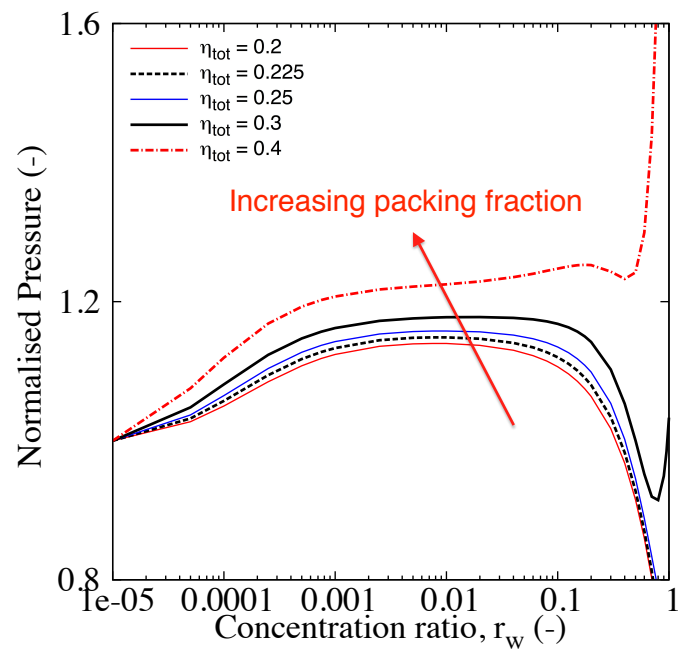

(a) Normalised pressure

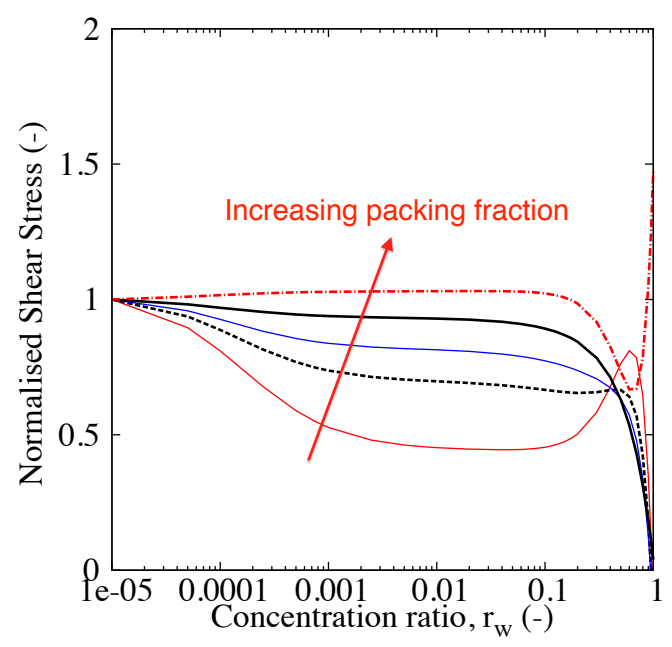

(b) Normalised shear stress

Figure 4. Variation in total packing fraction, $\eta_{\text {tot }}^{*}$ for $h s$ and 4-mer hc composition considering different concentration ratio, $r_{w}\left(\epsilon_{h c}=10, \epsilon_{h s}=0.1\right)$ 


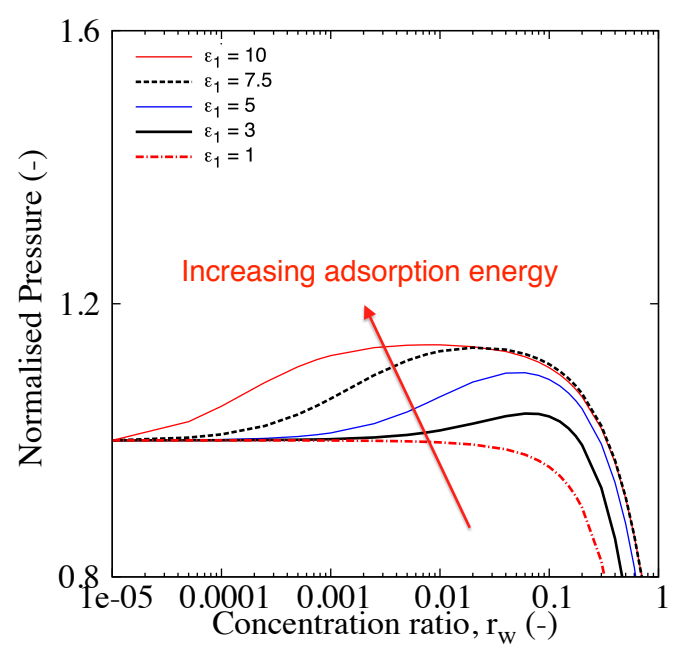

(a) Normalised pressure

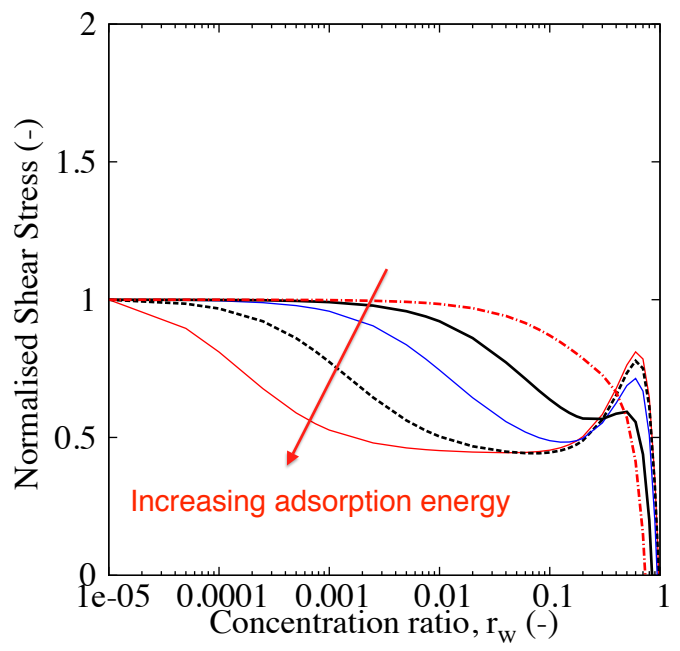

(b) Normalised shear stress

Figure 5. Variation in adsorption energy, $\epsilon_{h c}$ for $h s$ and 4-mer $h c$ composition considering different concentration ratio, $r_{w}\left(\eta_{t o t}^{*}=0.2, \epsilon_{h s}=0.1\right)$

Figure 6 shows that when progressively longer chain molecules are used the boundary shear stress is reduced because of a larger coverage of the conjunctional surfaces. It is noteworthy that the same adsorption energy is assumed for all the m-mer chains.

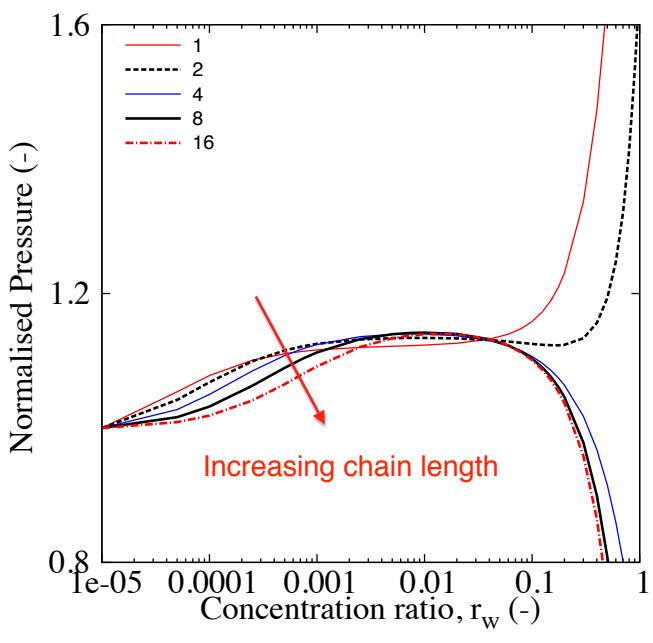

(a) Normalised pressure

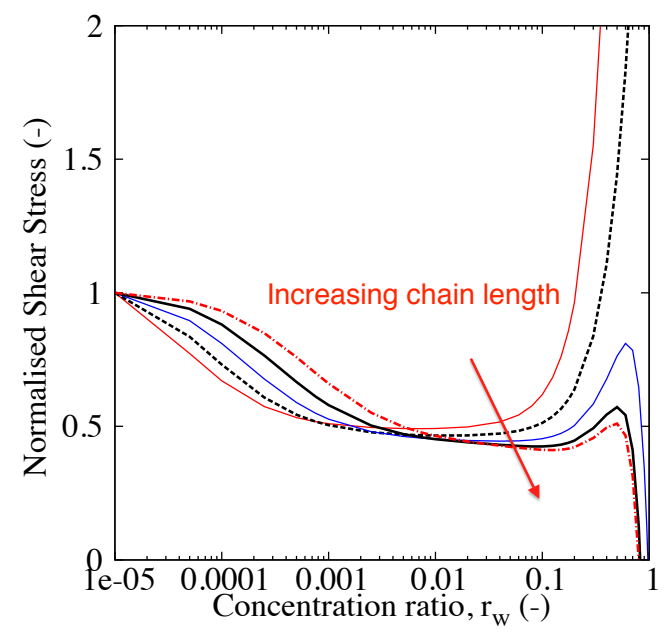

(b) Normalised shear stress

Figure 6. Variation in molecular chain lengths of hard chains in a hard sphere solvent $\left(\eta_{t o t}^{*}=0.2, \epsilon_{h c}=10, \epsilon_{h s}=0.1\right)$

\section{Conclusion}

The method described predicts characteristic behaviour of real physical fluids, pertinent to typical composition of lubricants. Base oil molecules here represented as spheres 
account for the load carrying capacity of the conjunction even in nano-scale, similar to their behaviour in micro-scale, except that in ultra-thin conjunctions the generated pressures are due to the solvation effect. The long chain molecules with "sticky" ends adsorb to the bounding solids, thus affect the shear characteristics of thin films. These findings are in accord with knowledge which has been accumulated through experience. Thus, the expounded approach is an affirmation of the current practice, but through a fundamental study. The analytical nature of the OZ-PY method and its application to boundary lubrication represents the main contribution of this paper, an approach which has not hitherto been reported in literature. This initial study should be expanded to include branched molecular chains and a larger species of molecules to better represent real lubricants. In particular, the inclusion of surfactants and anti-oxidants would affect the mechanism of adsorption of molecular species to the bounding surfaces. These observation constitute the future directions of the current research. Another important consideration is formulation of the method for application to micro-scale contact of macroscopic solids through its integration with statistical representation of rough surface topography.

\section{Acknowledgements}

The authors acknowledge the technical support and sponsorship provided by the EPSRC through the ENCYCLOPAEDIC program grant.

[1] G. Amontons. De la resistance cause e dans les machines. Mem. Acad. R. A, pages 275-282, 1699.

[2] C. A. Coulomb. Recherches sur la meilleure maniere de fabriquer les aigulles aimantes. Mem. Math. Phy. IX, pages 166-342, 1780.

[3] I. Newton. The mathematical papers of Isaac Newton. Vol. III: 1670 to 1673. Edited by D. T. Whiteside, with the assistance in publication of M. A. Hoskin and A. Prag. Cambridge University Press, London, 1969.

[4] C. L. M. H. Navier. Memoire sur les lois du mouvement des fluides. Mem. Acad. R. Sci, 6:389-440, 1823.

[5] S. D. Poisson. Memoire sur les equations generals de lequilibre et du movement des corps solids elastique et des fluids. J. Ecole Polytech, 13:1-174, 1831.

[6] G. G. Stokes. On the theories of the internal friction of fluids in motion. Trans. of Cambridge Phil. Soc., 8:287-319, 1845.

[7] O. Reynolds. On the theory of lubrication and its application to mr beauchamp tower's experiments, including an experimental determination of the viscosity of olive oil. Philosophical Transactions of the Royal Society, 177:157-234, 1886.

[8] H. Hertz. On the contact of elastic solids. J. reine angew. Math., 92:156-171, 1881.

[9] A. N. Grubin. Fundamentals of the hydrodynamic theory of lubrication of heavily loaded cylindrical surfaces. Central Scientific Research Institute for Technology and Mechanical Engineering, Moscow (DSRI Translation, No. 337), 1949.

[10] N. P. Petrov. Friction in Machines and the Effect of the Lubricant. Inzh. Zh., St. Peterb, 1883.

[11] W. B. Hardy and I. Doubleday. Boundary lubrication - the paraffin series. Proc. R. Soc. London, Ser. A, 100:550-574, 1921.

[12] D. Dowson. Thin films in tribology. In Proc. 19th Leeds-Lyon Symp. on Tribology, volume 25, pages 3-12, 1993.

[13] F. P. Bowden and D. Tabor. Mechanism of metallic friction. Nature, 150:197-199, 1942.

[14] J. N. Israelachvili. Intermolecular and surface forces. Academic press London, 1992. 
[15] D. Y. C. Chan and R. G. Horn. The drainage of thin liquid films between solid surfaces. J. Chem. Phy., 83(10):5311-5324, 1985.

[16] R. Gohar and H. Rahnejat. Fundamentals of tribology. Imperial College Press, London, 2008.

[17] H. Matsuoka and T. Kato. An ultrathin liquid film lubrication theory - calculation method of solvation pressure and its application to the ehl problem. J. Trib., 119:217-226, 1997.

[18] M. Al-Samieh and H. Rahnejat. Ultra-thin lubricating films under transient conditions. J. Phy. D: App. Phy., 34:2610-2621, 2001.

[19] M. F. Abd. Al-Samieh and H. Rahnejat. Nano-lubricant film formation due to combined elastohydrodynamics and surface force action under isothermal conditions. Proc. Inst. Mech. Eng., Part C : J. Mech. Eng. Sci., 215:1019-1029, 2001.

[20] M. Teodorescu, S. Balakrishnan, and H. Rahnejat. Physics of ultra-thin surface films on molecularly smooth surfaces. Proc. Inst. Mech Eng, Part N: J. Nanoeng and Nanosys, 220(1):719, 2006.

[21] M. Al-Samieh and H. Rahnejat. Physics of lubricated impact of a sphere in a plate in a narrow continuum to gaps of molecular dimensions. J. Phy. D: App. Phy., 35:2311-2326, 2002.

[22] W. W. F. Chong, M. Teodorescu, and H. Rahnejat. Effect of lubricant molecular rheology on formation and shear of ultra-thin surface films. J. Phy. D : App. Phy., 44(16):165302, 2011.

[23] W. W. F. Chong, M. Teodorescu, and H. Rahnejat. Formation of ultra-thin bi-molecular boundary adsorbed films. J. Phy. D : App. Phy. (Under-review), 2011.

[24] L. S. Ornstein and F. Zernike. Accidental deviations of density and opalescence at the critical point of a single substance. Proc. Roy. Acad., Amsterdam, 17:793-806, 1914.

[25] J. K. Percus and G. J. Yevick. Analysis of classical statistical mechanics by means of collective coordinates. Phy. Rev., 110:1-13, 1958.

[26] M. S. Wertheim. Fluids with highly directional attractive forces. part 1 - statistical thermodynamics. J. Stat. Phy., 35:19, 1984.

[27] M. S. Wertheim. Fluids with highly directional attractive forces. part 2 - thermodynamic perturbation theory and integral equations. J. Stat. Phy., 35:35, 1984.

[28] M. S. Wertheim. Fluids with highly directional attractive forces. part 3 - multiple attraction sites. J. Stat. Phy., 42:459, 1986.

[29] M. S. Wertheim. Fluids with highly directional attractive forces. part 4 - equilibrium polymerization. J. Stat. Phy., 42:477, 1986.

[30] J. Chang and S. I. Sandler. The correlation functions of hard-sphere chain fluids: Comparison of the wertheim integral equation theory with the monte carlo simulation. J. Chem. Phy., 102(1):437-449, 1994.

[31] N. Wu and Y. C. Chiew. Multidensity integral-equation theory for short diblock hard-spheresticky-hard-sphere chains. Phy. Rev. E, 81:041809, 2010.

[32] R. J. Baxter. Percus-yevick equation for hard spheres with surface adhesion. J. Chem. Phy., 49(6):2770-2774, 1968.

[33] J. W. Perram and E. R. Smith. A model for the examination of phase behaviour in multicomponent systems. Chem. Phy. Lett., 35(1):138-140, 1975.

[34] Y. C. Chiew. Percus-yevick integral equation theory for thermal hard-sphere chains- part 1: Equations of state. Mol. Phy., 70(1):129-143, 1990.

[35] Y. C. Chiew. Percus-yevick integral equation theory for thermal hard-sphere chains- part 2: Average intermolecular correlation functions. Mol. Phy., 73(2):359-373, 1990.

[36] Y. C. Chiew. Intermolecular site-site correlation functions of thermal hard-sphere chains: Analytic integral equation theory. J. Chem. Phy., 93(7):5067-5074, 1990.

[37] P. Attard and J. L. Parker. Oscillatory solvation forces: A comparison of theory and experiment. J. Phy. Chem., 96(12):5086-5093, 1992.

[38] B. V. Derjaguin. Adhesion of small particles. Kolloid-Zeit., 69:155-164, 1935.

[39] F. Hirata. Molecular Theory of Solvation. Kluwer Academic Publishers, Dordrecht, 2003.

[40] J. W. Perram and E. R. Smith. Percus-yevick theory of adsorption. Chem.Phy. Lett., 39(2):328- 
$332,1976$.

[41] R. J. Baxter. Ornstein-zernike relation and percus-yevick approximation for fluid mixtures. J. Chem. Phy., 52(9):4559-4562, 1970.

[42] M. S. Wertheim. Analytic solution of the percus-yevick equation. J. Math. Phy., 5:643-651, 1964.

[43] J. W. Perram and E. R. Smith. Competitive adsorption via percus-yevick approximation. Proc. Roy. Soc. A, 353:193-220, 1977.

[44] P. T. Cummings, J. W. Perram, and E. R. Smith. Percus-yevick theory of correlation functions and nucleation effects in the sticky hard-sphere model. Mol. Phy., 31(2):535-548, 1976.

[45] H. Eyring. Viscosity, plasticity, and diffusion as examples of absolute reaction rates. J. Chem. Phy., 4:283, 1936.

[46] M. He, A. Szuchmacher Blum, G. Overney, and R. M. Overney. Effect of interfacial liquid structuring on the coherence length in nanolubrication. Phy. Rev. Lett., 88(15):154302, 2002.

\section{Appendix: Nomenclature}

$E_{y} \quad$ Barrier height for Eyring model $(J)$

$N \quad$ Number of molecule species (-)

$N_{c} \quad$ Number of chain molecules (-)

$Q_{y} \quad$ Process activation energy $(J)$

$R_{e f f} \quad$ Effective particle diameter $(m)$

$R_{\alpha \beta} \quad$ Reduced particle diameter, $\left(\sigma_{\alpha}+\sigma_{\beta}\right) / 2,(m)$

$S_{\alpha \beta} \quad$ Average diameter difference between interacting particles, $\left(\sigma_{\alpha}-\sigma_{\beta}\right) / 2,(m)$

$T \quad$ Temperature of the fluid system $\left({ }^{\circ} \mathrm{C}\right)$

$\bar{T} \quad$ Dimensionless temperature of the fluid system $(-)$

$V \quad$ Total volume of the system $\left(\mathrm{m}^{3}\right)$

$c^{*} \quad$ Indirect correlation function $(-)$

$d_{\alpha} \quad$ Adsorption parameter for particle species $\alpha(-)$

$g^{*} \quad$ Pair correlation function (-)

$h^{*} \quad$ Direct correlation function $(-)$

$h c \quad$ Hard chains $(-)$

hs $\quad$ Hard sphere $(-)$

$k_{B} \quad$ Boltzmann constant $\left(\mathrm{m}^{2} \mathrm{kgs}^{-2} \mathrm{~K}^{-1}\right)$

$m \quad$ Chain length $(-)$

$p_{s} \quad$ Solvation pressure $(\mathrm{Pa})$

$q_{\alpha \beta} \quad$ Parameter for transformed Ornstein-Zernike (OZ) equation (-)

$r^{*} \quad$ Distance between the centre of two particles $(m)$

$s \quad$ Distance between the centre of a particle towards a reference particle $(m)$

$t \quad$ Time step $(s)$

$\Delta \quad$ Step size (-)

$\Omega_{y} \quad$ Lubricant volume where pressure, $p$ is acting on $\left(m^{3}\right)$

$\alpha, \beta, \gamma \quad$ Particle species (-)

$\delta \quad$ Dirac delta function $(-)$

$\epsilon \quad$ Adsorption energy (-)

$\eta^{*} \quad$ Particle packing fraction $(-)$

$\eta^{o} \quad$ Packing fraction for the particle forming the chain (-)

$\lambda_{\alpha \beta} \quad$ Adsorption-temperature relation for the "sticky" hard sphere particle (-)

$\lambda_{\alpha \beta}^{o} \quad$ Adsorption-temperature relation for the particle forming the chain molecule (-)

$\phi_{\alpha \beta} \quad$ Interaction potential between particles (-)

$\phi_{y}^{*} \quad$ Activation volume $\left(m^{3}\right)$

$\rho^{*} \quad$ Density number of a particle $(-)$

$\rho_{c}^{*} \quad$ Density number of a chain molecule $(-)$ 
$\rho^{o} \quad$ Density number of a particle forming the chain molecule (-)

$\sigma \quad$ Particle diameter $(m)$

$\tau_{y} \quad$ Shear stress $(\mathrm{Pa})$

$\zeta_{i} \quad$ Parameter for variable $q_{\alpha \beta}$ where $i=1,2$, and 3 (-) 